\title{
REVIEW
}

\section{A Systematic Review of Assessment Tools Measuring Cultural Competence Outcomes Relevant to Pharmacy Education}

\author{
Melissa Medina, EdD, ${ }^{\mathrm{a}}$ Jaime Maerten-Rivera, PhD, ${ }^{\mathrm{b}}$ Yichen Zhao, ${ }^{\mathrm{b}}$ Brianna Henson, MPA ${ }^{\mathrm{c}}$ \\ ${ }^{\text {a }}$ The University of Oklahoma, University of Oklahoma Health Sciences Center, Oklahoma City, Oklahoma \\ ${ }^{\mathrm{b}}$ University of Buffalo, School of Pharmacy and Pharmaceutical Sciences, Buffalo, New York \\ ${ }^{\mathrm{c}}$ Equus Workforce Solutions, Louisville, Kentucky
}

Corresponding Author: Melissa S. Medina, The University of Oklahoma, College of Pharmacy, 1110 N Stonewall Ave., Oklahoma City, OK 73117. Tel: 405-271-6484. Email: Melissa-medina@ouhsc.edu

Submitted April 1, 2021; accepted January 2, 2022; ePublished January 2022

Objective. To identify and describe validated assessment tools measuring cultural competence relevant to pharmacy education.

Methods. A systematic approach was used to identify quantitative cultural competence assessment tools relevant to pharmacy education. A systematic search of the literature was conducted using the OVID and EBSCO databases and a manual search of journals deemed likely to include tools relevant to pharmacy education. To be eligible for the review, the tools had to be developed using a study sample from the US, have at least one peer-reviewed validated publication, be applicable to the pharmacy profession, and be published since 2010 .

Results. The search identified a total of 27 tools from the systematic literature and manual search. A total of 12 assessment tools met the criteria to be included in the summary and their relevancy to pharmacy education is discussed. Conclusion. A review of literature demonstrates that assessment tools vary widely and there is no universal tool to assess cultural competence in pharmacy education. As cultural competence is a priority within the accreditation standards for Doctor of Pharmacy education, pharmacy programs are encouraged to develop additional tools that measure observed performance.

Keywords: programmatic assessment, academic assessment, cultural competence, cultural sensitivity

\section{INTRODUCTION}

Cultural competence (CC) is an important program outcome in pharmacy education because pharmacists who understand how cultural beliefs influence patients' health, illness, medication taking behaviors, and treatment decisions and consider these beliefs when providing care, are thought to practice in a culturally competent manner. ${ }^{1}$ Culture includes race, ethnicity, socioeconomic status, religion, age, gender, sexual orientation, disability, and health beliefs. ${ }^{1}$ Cultural competency is an ongoing process that does not have a fixed endpoint. CC refers to an ability to understand and effectively communicate with people across cultures and it includes: 1) awareness of one's own cultural beliefs, 2) attitude towards different cultures, 3) knowledge of different cultural practices, and 4) cross-cultural skills. ${ }^{1-4}$ In health care, CC includes a set of congruent behaviors, knowledge, and attitudes among professionals that allows for the provision of effective care to meet patients' culturally unique needs. ${ }^{1}$ Relatedly, health disparities are preventable differences that are experienced by socially disadvantaged populations that create barriers to achieving optimal health outcomes. ${ }^{5}$ Negative health outcomes may result when sociocultural differences between patients and providers are incompatible. ${ }^{6}$ Research demonstrates that there is a clear link between cultural competent care and eliminating racial/ethnic disparities in health care. ${ }^{6}$ Sometimes cultural sensitivity is used in lieu of CC in pharmacy education since it is impossible for anyone to be competent in all cultures and it is easier to capture the level of knowledge, skills, and attitudes that students possess upon graduatiton ${ }^{7-9}$ However, CC adds in a level of operability by encouraging providers to reflect upon their beliefs, engage patients, and tailor health care delivery to individual needs. ${ }^{1-4}$ For consistency, $\mathrm{CC}$ is the most common term and it will be used in this review.

There is growing emphasis on teaching and assessing $\mathrm{CC}$ in pharmacy education and integrating it across the entire curriculum (didactic, experiential, and co-curriculum) as documented in the release of the 2013 Center for Advancement of Pharmacy Education Outcomes (CAPE) that lists it as one of the 15 outcomes expected of pharmacy students upon graduation. ${ }^{7}$ The 2016 Accreditation Council for Pharmacy Education (ACPE) Standards require pharmacy programs to teach and assess CC and cultural sensitivity in both the didactic and experimental curricula so that students 
have the ability to practice in culturally diverse environments. ${ }^{9}$ Pharmacy students need specific training in their curriculum so they can better understand their own and their patients' health care beliefs, recognize when health disparities exist, and deliver patient care in a culturally sensitive manner so they can provide the best care for their patients. ${ }^{1}$

While pharmacy programs are including more CC content into their curriculum, the assessment of these outcomes lags behind and no best practices exist. ${ }^{1,10}$ Multiple manuscripts urge for integration of these topics in pharmacy but there is uncertainty because there is not a comprehensive national assessment to how this content should be incorporated. ${ }^{11}$ This is a concern amongst other health profession programs such as nursing and medicine, where there is a lack of consensus on how this content should be structured, organized, and facilitated. ${ }^{12}$ A previous White Paper outlined individual and programmatic assessment tools that were used in health care education (ie, colleges and schools of pharmacy, medicine, occupational therapy, physical therapy, and nursing) as well as in health care practice areas such as hospitals and general counseling settings, however, this work is approximately eight years old. ${ }^{2}$ Therefore, the goal of this review is to update the existing literature by identifying and evaluating the available validated tools for measuring CC in pharmacy students.

\section{METHODS}

Systematic literature review was used to identify CC assessment tools with published psychometric information published since 2010 that would be relevant for use in pharmacy education. As a first step in the systematic review, to identify studies, a full text publication search was conducted using the OVID and EBSCO databases. The relevant terms used in this search were: cultural competence, multiculturalism, cross-cultural, intercultural, cultural sensitivity, cultural empathy, health disparities, and diversity education. Next, a manual search of pharmacy education journals (ie, American Journal of Pharmaceutical Education, Currents in Pharmacy Teaching and Learning, Pharmacy Education, Journal of the American College of Clinical Pharmacy, American Journal of Health System Pharmacy) was conducted using the same relevant terms.

In order to select studies for the next step of the systematic review, the inclusion and exclusion criteria of article selection were discussed and determined by the authors prior to the initial literature search. Articles were collected if they: (1) were empirical and peer-reviewed; (2) developed, validated, or applied measures of cultural competence using a study sample from the US; (3) focused on health care professions (eg, pharmacy, nursing, medicine, dental, physical therapy, etc.); (4) were recently published (2010 and after). Two rounds of literature search were conducted. In the first round of search, the abstracts of the publications retrieved from the search, were reviewed to determine if the publication developed or applied a CC assessment tool, and only publications meeting this criterion were retained. Next, all the databases were searched again to ensure that no relevant publications were missed. Some publications used a CC assessment tool and cited relevant psychometric publications or research publications that used the tool; these publications were retrieved in order to gather all relevant information on the assessment tool and its use. That is, if an assessment tool was applied by a recent research since 2010, the previous studies that developed and validated this assessment tool were also collected during the second round of search. These two steps were conducted by one author (YZ) and if an article was questionable for inclusion at these steps, a second author (JMR) was consulted for a second review and discussion until consensus occurred.

For the third step of the systematic review, evaluating the identified articles, all authors of this study reviewed the publications related to the remaining tools and conducted a critical appraisal, which included a discussion of strengths and weaknesses of the measures, and the applicability to pharmacy education. The criteria used to assess the tools included determining if the tool: (1) measured CC, (2) had at least one peer-reviewed publication examining its development and reliability and items were accessible for review, (3) was applicable to pharmacists or pharmacy students (ie, the tool language or questions did not preclude pharmacists/student pharmacists; did not assess a practice situation irrelevant to pharmacists, or did not require significant question revision to be applicable for pharmacy).

For tools that were reviewed and met all criteria, key information was presented in a descriptive summary table. The descriptive information included the following: name, overview, item description, and relevant validation and research studies.

\section{RESULTS}

The initial publication search for a developed tool or evaluation of a relevant tool identified 72 articles, covering 36 measures, for further review. Some tools had undergone revision; in such cases, the most recent version was retained. Nine articles did not provide names for measures, did not contain measure information, or were not peer reviewed and removed from review. This identified 63 articles ( 27 measures) for further review. Of these 27 tools, six were excluded because the study sample was not from the US leaving 21 tools for critical appraisal by the study authors. Of these 21 studies, two tools were excluded because they did not measure CC (one measured empathy and the other hospital 
systems); three were excluded because there was insufficient information on the development and reliability (ie, the tool was researcher-developed for the specific study); and four were excluded because they were not applicable to pharmacists/pharmacy students. In the end, there were 30 articles examining 12 assessment tools that met inclusion criteria. Figure 1 contains the $\mathrm{CC}$ tool publication search and selection schematic. The key information for these tools is presented in Table 1.

In Table 1, the authors identified three main categories of tools based on their evaluation of the article, purpose of the measure, the background of the students the tool was used in (e.g., first year or APPE students), and the tool questions. This resulted in three tool grouping categories. The first category covered tools designed to be used for students with health care practice experience. There were seven tools grouped into this area. These tools generally contained at least some questions that asked about knowledge or skills used while providing patient care. For example, the Clinical Cultural Competency Questionnaire (CCCQ) assesses the skills domain with questions like "providing culturally competent patient education and counseling", which incoming pharmacy students would not be able to respond to. ${ }^{13}$ However, more advanced students would be able to assess this as they have clinical experience.

The second category covered tools designed for broad student use. There were four tools grouped into this area. These tools are not specific to pharmacy or health care, yet many have been used with health care or pharmacy students, particularly those early on in their respective programs (ie, professional year 1 pharmacy students). An example of this is the Everyday Multicultural Competencies/Revised SEE (EMC/RSEE) which was developed from four existing measures (see Table 1). ${ }^{14}$ It assesses CC areas and items including cultural openness and desire to learn (example of item "A truly good education requires knowing how to communicate with someone from another culture") and awareness of contemporary racism and privilege (example of item "I am aware of how society differentially treats racial and ethnic groups other than my own").

The final category in Table 1 covered tools designed for curriculum assessment and included only one measure, the Revised Tool for Assessing Cultural Competence Training (TACCT-R). ${ }^{15}$ This measure was initially designed to help medical colleges integrate $\mathrm{CC}$ content in the curriculum and used a panel of experts to identify relevant areas for training. The instrument covers topics including knowledge, skills, and attitudes impacting CC training on six domains. The instrument has been adapted for use in nursing and dental, and the domains were recently used as a framework in a pharmacy study. ${ }^{16-18}$ This type of instrument can be useful to identify under-addressed curriculum areas, measure faculty and student perception of the curriculum, and to monitor new curriculum innovations.

\section{DISCUSSION}

The results of this review identified $12 \mathrm{CC}$ tools that met inclusion criteria. These 12 tools were categorized into three main areas of pharmacy education including seven tools for use with students with health care practice experience, four tools for broad student use, and one tool for evaluating the curriculum. The number of questions on the tools ranged from 15 to 75 and most used subscales or domains within the tools (range of two to six subscales or domains). Most of the tools were free and the scales and items were available for review in the publications. However, one tool, the IAPCC-R, had a fee for use. ${ }^{19}$ Some tools used published a validation study which included pharmacy students. 19 While other tools had been used in research with pharmacy students evidencing their applicability. ${ }^{18,20-30}$ Additionally, some tools had been validated or used with health care professionals or health care professional students. ${ }^{15,19,27,31-37}$ Only one tool was identified that assessed cultural competence in the curriculum. ${ }^{15,18,30}$ However, this was included as it met the criteria for study inclusion and future efforts may encourage programs to fully delineate cultural competence outcomes and map those outcomes in their overall assessment plan for their didactic and experiential curriculum and co-curriculum, similar to work done in interprofessional education thus it may be useful to programs. ${ }^{38}$ Others have stressed that it is important for programs to develop an integrated program of assessment and adhere to best practices for assessment in general, and this is true regarding CC. ${ }^{39,40}$ The CC integrated program of assessment should consider the who, what, where, when, why as answers to these questions will help in selecting an appropriate tool.

The first consideration is who are the students being assessed (ie, first professional year students versus students with practice experience). The measures reviewed in the current study are grouped according to who would be able to respond to most questions (ie, students with health care experience versus tools for broad student use). Addressing who is completing the assessment will assist schools/colleges of pharmacy in finding the most relevant and appropriate tool(s). Next, what is desired to be measured; most of the tools identified in this review are readily available and items can be reviewed within the reference's publications. Programs should evaluate the tool items and subscales to ensure that they align with their intended CC learning objectives/outcomes. The decision of where in the program to assess students is important and may be driven by where in the student experience self-awareness or growth may be triggered. Based on this information programs must decide when to assess students so they can determine if students' CC perceptions improve over time. Programs should consider assessing students at baseline, or early in the curriculum, so they can track changes 
over time. Decisions will need to be made regarding the exact times to conduct follow-up. For example, programs may want to reassess students after they have been exposed to or completed specific experiences so the effectiveness of a specific event(s) (ie, instruction on topic, experience) can be evaluated. Alternatively, a program may want to examine change or growth across multiple years (ie, $\mathrm{CC}$ instruction intentionally layered within several courses) and therefore a baseline with annual follow-ups may be administered.

There are three limitations to our study. First, the tools identified in this review measured students' CC, but tools for use in other areas that may be related to $\mathrm{CC}$, such as empathy and cultural humility were not identified for review. This was to keep the focus on a specific area of tools that are most relevant to programs developing a CC assessment plan. Second, the review process may have not located all cultural competence tools relevant to pharmacy education. We conducted an extensive search and review; however, tools are continuously being developed and we may have failed to identify tools that may have been relevant, particularly if the publication(s) was outside of the pharmacy area. Similarly, we attempted to identify relevant research publications that used the tools as this provides additional information on how the tools have been used; however, we may have failed to identify some publications in our search. A strength of our study is that the authors strictly adhered to the inclusion criteria of selecting articles and tools and vetted all of the tools identified in the review as a team, including robust discussion leading to consensus. Future research should repeat this review process as the field will continue to expand as existing tools are modified and new tools are developed to improve instruction and assessment of CC.

\section{Future Directions for Assessing CC}

There are five additional considerations when selecting tools to include in a CC assessment plan. First, all of the tools identified in our review were quantitative, self-report measures designed to assess the perceptions of the person completing the tool versus his/her authentic behaviors and actions with people of different cultures. Adding assessments of observed performance is important since perceptions of competence does not guarantee that people will use their CC skills appropriately. Therefore, utilizing a mixture of both quantitative and observation, reflective writing and/or qualitative tools may provide a more complete picture of those being assessed. ${ }^{38}$ For example, tools such as culture clinical case vignettes or objective structured clinical examinations (OSCEs) could be used alone or with quantitative tools to assess patient-centered culturally sensitive health care knowledge and skills. ${ }^{41}$ Those pursuing this mixture of assessments can consult the literature for recommendations. ${ }^{6,42}$ Future tool development and research may include developing tools that evaluate culturally congruent clinical practice and/or include assessments of how students/practitioners actually act, not just their self-assessed knowledge/skills/behaviors. ${ }^{43}$

Second, when selecting tools to include in a CC assessment plan, programs should examine the subscales/domains and the individual items on the assessment for relevancy. For example, the CCCQ, CCHPA-67, and HPCCI tools contained some questions that were focused on physicians and not applicable to pharmacy practice. ${ }^{31,33}$ These questions would need to be modified if the tool was selected for use with pharmacy students, which was done with the CCCQ. ${ }^{13}$ In addition, the number of questions in the tool the selected tool(s) should be assessed for the possibility of survey fatigue as some of the tools found in this review contained up to 75 items. It should be noted that some of the subscales/domains may be relevant to an assessment while others may be less relevant. None of the studies mentioned whether selected subscales or tools could be used without using the entire tool. Yet this may be one way to reduce the number of items while accomplishing a robust assessment of relevant areas, particularly if different assessments are combined (ie, broad use tool to assess a student's perspectives combined with tool for students with health care practice experience to assess a student's practice behavior). Another possibility for combining assessments would be to use a tool for broad student use as students enter the program and/or early in the program, then when students enter their IPPE or APPE years, supplement or replace the assessment with a tool one for use with students with health care experience (See Table 1). It is important to note that if programs modify a tool to fit their need, adding or deleting questions may change the psychometric properties of the tool.

Next, most publications identified in the review assessed participants at one timepoint and a few publications assessed students at pre/post (CCCQ, CCHPA, EMC/RSEE, IAPCC, SEE). ${ }^{13,18,20,24-26,32,34,36}$ None of the studies of these tools had been used longitudinally beyond pre/post to assess growth. Although measures may be effective at measuring $\mathrm{CC}$ at one point in time, evidence of being sensitive enough to detect change or growth in CC requires additional study. Programs should consider this when selecting a tool.

Fourth, when determining if a tool should be included in a CC assessment plan, attention should be placed on the number, recency, and generalizability of research publications pertaining to the tool. For example, the EMC/RSEE, the ISS, and the MEQ may be appealing tools to use to assess pharmacy students early in the curriculum and the CCA later with students when they gain health care experience, but only one study was published for each tool. ${ }^{29,34,35,37}$ Reviewing the published literature can also provide insights on how a programs is modifying a tool(s). ${ }^{44}$ The IAPCC is a tool that has 
been used frequently in the literature and it has a student version, but the fee for use may prohibit some programs from adopting it if financial resources are not available. ${ }^{18,24-26}$

Finally, while the quantitative tools identified in the review assessed the student or practitioner's cultural competence or sensitivity, none assessed perceptions or practices for how the student or practitioner responds to receiving culturally inappropriate messages or behaviors. Students were also not allowed to explain their choices or the context behind their answer choice. Future tools and studies should include assessments of how to respond to cultural incompetence, prejudice or bias. ${ }^{45-47}$

\section{CONCLUSION}

Standards 2016 emphasize the importance of CC instruction and assessment. This study used a systematic process to identify $12 \mathrm{CC}$ quantitative assessment tools relevant to pharmacy education. The tools were grouped by categories according to how they can be used: tools for students with health care practice experience, tools for broad student use, and tools for curriculum assessment. There are a number of considerations when choosing a CC assessment tool and developing a CC assessment plan, including the use of multiple tools at different points. The 12 tools in this review all measure self-assessment of CC. The field would benefit from the development of additional tools that measure observed performance.

\section{REFERENCES}

1. O'Connell MB, Korner EJ, Rickles NM, Sias JJ. Cultural competence in health care and its implications for pharmacy part 1: Overview of key concepts in multicultural health care. Pharmacotherapy. 2007;27(7):10621079.

2. O'Connell MB, Rodriguez de Bittner M, Poirier T, et al. Cultural competency in health care and its implications for pharmacy part 3A: Emphasis on pharmacy education, curriculums, and future directions. Pharmacotherapy. 2013;33(12):e347-e367.

3. National Association of Social Workers. Standards and Indicators for Cultural Competence in Social Worker Practice. 2015 . https://www.socialworkers.org/LinkClick.aspx?fileticket=7dVckZAYUmk\%3D\&portalid=0. Accessed December 1, 2021.

4. Flaskerud JH. Cultural competence column what else is necessary? Issues Ment Health Nurs. 2007;28(2):219222.

5. Centers for Disease Control and Prevention, U.S. Department of Health and Human Services Health Disparities Among Youth. https://www.cdc.gov/healthyyouth/disparities/index.htm. Accessed December 1, 2021.

6. Betancourt JR, Cervantes MC. Cross-cultural medical education in the united states: Key principles and experiences. Kaohsiung J Med Sci. 2009;25(9):471-478.

7. Medina MS, Plaza CM, Stowe CD, et al. Center for the Advancement of Pharmacy Education 2013 educational outcomes. Am J Pharm Educ. 2013;77(8):Article 162.

8. Kamaka ML. Designing a cultural competency curriculum: Asking the stakeholders. Hawaii Med J. 2010;69(6 Suppl 3):31-34.

9. Accreditation Council for Pharmacy Education. Accreditation Standards and Key Elements for the Professional Program in Pharmacy Leading to the Doctor of Pharmacy Degree ("Standards 2016"). Published 2015. https://www.acpe-accredit.org/pdf/Standards2016FINAL.pdf. Accessed December 1, 2021.

10. O'Connell MB, Rickles NM, Sias JJ, Korner EJ. Cultural competence in health care and its implications for pharmacy part 2: Emphasis on pharmacy systems and practice. Pharmacotherapy. 2009;29(3): 14e-34e .

11. Chen AMH, Armbruster AL, Buckley B, et al. Inclusion of health disparities, cultural competence, and health literacy content in US and Canadian pharmacy curriculums. Am J Pharm Educ. 2021;85(1):Article 8200.

12. O'Brien EM, O’Donnell C, Murphy J, O'Brien B, Markey K. Intercultural readiness of nursing students: An integrative review of evidence examining cultural competence educational interventions. Nurs Educ in Prac. 2021;50:102966.

13. Echeverri M, Brookover C, Kennedy K. Nine constructs of cultural competence for curriculum development. Am J Pharm Educ. 2010;74(10):Article 181.

14. Mallinckrodt B, Miles JR, Bhaskar T, Chery N, Choi G, Sung MR. Developing a comprehensive scale to assess college multicultural programming. J Couns Psychol. 2014;61(1):133-145.

15. Lie DA, Boker J, Crandall S, et al. Revising the Tool for Assessing Cultural Competence Training (TACCT) for curriculum evaluation: Findings derived from seven US schools and expert consensus. Med Educ Online. 2008;13(1):11. 
16. Watts RJ, Cuellar NG, O'Sullivan AL. Developing a blueprint for cultural competence education at Penn. J Prof Nurs. 2008;24(3):136-142.

17. Holyfield LJ, Miller BH. A tool for assessing cultural competence training in dental education. J Dent Educ. 2013;77(8):990-997.

18. Chen AMH, Cailor SM, Wicker E, Harper NG, Franz TT, Pahl B. Integrating health literacy and cultural competency concepts across the doctor of pharmacy curriculum. Am J Pharm Educ. 2020;84(10):Article 764.

19. Transcultural CARE Associates. Inventory for Assessing the Process of Cultural Competence Among Healthcare Professionals-Student Version (IAPCC-SV). http://transculturalcare.net/iapcc-sv/. Accessed December 1, 2021.

20. Butler L, Chen AMH, Borja-Hart N, et al. Assessment of a multi-institution integration of cultural competency activities. Curr Pharm Teach Learn. 2020;12(5):517-523.

21. Crawford SY, Awé C, Tawk RH, Pickard AS. A cross sectional and longitudinal study of pharmacy student perceptions of readiness to serve diverse populations. Am J Pharm Educ. 2016;80(4):Article 62.

22. Okoro ON, Odedina FT, Reams RR, Smith WT. Clinical cultural competency and knowledge of health disparities among pharmacy students. Am J Pharm Educ. 2012;76(3):Article 40.

23. Okoro O, Odedina F, Smith WT. Determining the sufficiency of cultural competence instruction in pharmacy school curriculum. Am J Pharm Educ. 2015;79(4):Article 50.

24. Werremeyer AB, Skoy ET. A medical mission to Guatemala as an advanced pharmacy practice experience. Am $J$ Pharm Educ. 2012;76(8):Article 156.

25. Cailor SM, Chen AMH. Immediate and longitudinal effects of incorporating health literacy and cultural competency into a yearlong pharmacy curriculum. Curr Pharm Teach Learn. 2015;7(3):292-301.

26. Skoy E, Werremeyer A. Comparing photovoice to traditional reflection to identify student learning on a medical mission trip. Am J Pharm Educ. 2020;84(4):Article 7599.

27. Echeverri M, Dise T. Racial dynamics and cultural competence training in medical and pharmacy education. $J$ Health Care Poor Underserved. 2017;28(1):266-278.

28. Echeverri M, Brookover C, Kennedy K. Assessing pharmacy students' self-perception of cultural competence. $J$ Health Care Poor Underserved. 2013;24(1):64-92.

29. Ekong G, Kavookjian J, Hutchison A. Predisposition for empathy, intercultural sensitivity, and intentions for using motivational interviewing in first year pharmacy students. Am J Pharm Educ. 2017;81(8):Article 5989.

30. Aspden T, Butler R, Heinrich F, Harwood M, Sheridan J. Identifying key elements of cultural competence to incorporate into a New Zealand undergraduate pharmacy curriculum. Pharm Educ. 2017;17(1):43-54.

31. Harris-Haywood S, Goode T, Gao Y, et al. Psychometric evaluation of a cultural competency assessment instrument for health professionals. Med Care. 2014;52(2):e7-e15.

32. Mu K, Peck K, Jensen L, Bracciano A, Carrico C, Feldhacker D. CHIP: Facilitating interprofessional and culturally competent patient care through experiential learning in China. Occup Ther Int. 2016;23(4):328-337.

33. Schwarz JL, Witte R, Sellers SL, et al. Development and psychometric assessment of the healthcare provider cultural competence instrument. Inquiry. 2015;52:0046958015583696.

34. Kanter JW, Rosen DC, Manbeck KE, et al. Addressing microaggressions in racially charged patient-provider interactions: a pilot randomized trial. BMC Med Educ. 2020;20(1):88.

35. Domenech Rodríguez MM, Phelps PB, Tarp HC. Baseline cultural competence in physician assistant students. PLoS ONE. 2019;14(4): e0215910.

36. Fleming BD, Thomas SE, Burnham WS, Charles LT, Shaw D. Improving ethnocultural empathy in healthcare students through a targeted intervention. J Cult Divers. 2015;22(2):59-63.

37. Shah BK, Lonie JM. Assessing the cultural competency of practicing community pharmacists: a pilot study. Curr Pharm Teach Learn. 2012;4(4):240-246.

38. Shrader S, Farland MZ, Danielson J, Sicat B, Umland EM. A systematic review of assessment tools measuring interprofessional education outcomes relevant to pharmacy education. Am J Pharm Educ. 2017;81(6):Article 119.

39. Fielding DW, Regehr G. A call for an integrated program of assessment. Am J Pharm Educ. 2017;81(4):Article 77.

40. Poirier TI, Wilhelm M. Scholarly and best practices in assessment. Am J Pharm Educ. 2018;82(3):Article 6769.

41. Mihalic AP, Morrow JB, Long RB, Dobbie AE. A validated cultural competence curriculum for US pediatric clerkships. Patient Educ Couns. 2010;79(1):77-82.

42. Hamilton J. Intercultural competence in medical education - essential to acquire, difficult to assess. Med Teach. 2009;31(9):862-865.

43. Purnell L. Are we really measuring cultural competence? Nurs Sci Q. 2016;29(2):124-127. 
44. Echeverri M, Brookover C, Kennedy K. Factor analysis of a modified version of the California Brief Multicultural Competence Scale with minority pharmacy students. Adv Health Sci Educ Theory Pract. 2011;16(5):609-626.

45. Jain SH. The racist patient. Ann Intern Med. 2013;158(8):632.

46. Chandrashekar P, Jaime SH, Humphrey HJ. Racist patients: Taking action on harmful bias and discrimination in clinical learning environments. Presented at: The Josiah Macy Jr Foundation: Macy Conference 2021. https://bit.ly/3pUZH9D. Accessed December 1, 2021.

47. Cooper LA, Vellurattil RP, Quiñones-Boex A. Pharmacy students' perceptions of cultural competence encounters during practice experiences. Am J Pharm Educ. 2014;78(2):Article 31.

48. Echeverri M, Unni E, Harpe SE, et al. A multi-school validation of a revised scale for assessing cultural competence in pharmacy students. Am J Pharm Educ. 2019;83(3):Article 6602.

49. Chen GM, Starosta W. The development and validation of the intercultural sensitivity scale. Hum Commun. 2000;3:1-15.

50. Narvaez D, Hill PL. The relation of multicultural experiences to moral judgment and mindsets. J Divers High Educ. 2010;3(1):43-55. 
Table 1. Examples of Cultural Competence Assessment Tools

\begin{tabular}{llcl}
\hline Name & Overview & Item description & Validation and Research \\
Studies & & \\
\hline
\end{tabular}

Assessment Tools for Students with Healthcare Practice Experience

California Brief Multicultural Modified version of the California

Competency Scale (CBMCS) Brief Multicultural Competence

Modified

Scale (CBMCS), used to measure multicultural competence. Some of the questions use the wording "my patient" thus this would need to be used by students/practitioners seeing patients.

Cultural Competence

Assessment (CCA)

Cultural Competence Health Practitioner Assessment (CCHPA-67)

Healthcare Provider Cultural

Competence Instrument (HPCCI)

Clinical Cultural Competency Questionnaire (CCCQ)

Inventory for Assessing the Process of Cultural

Competence Among

\section{Assesses cultural competence among healthcare providers.}

Assess levels of cultural and linguistic competence of healthcare practitioners. Developed from 129 items Cultural Competence Health Practitioner Assessment (CCHPA129)

Assess knowledge of health disparities and various aspects of cultural competence. Some of the questions ask about patients or patient care this this would need to be used by students/practitioners seeing patients.

Measures the cultural competence of health care providers and can provide useful professional feedback for practitioners and organizations.

Measures level of cultural competence in healthcare delivery. Revised version of Inventory for
Self-assessment

22 items with 4 subscales (5-point Likert scale):

Assessing population health needs ( 7 items)

Multicultural knowledge (7 items)

Barriers to health care (8 items)

Sensitivity and responsiveness to consumers (3

items)

Self-assessment

25 items with 2 subscales (5-point Likert scale)

Cultural awareness and sensitivity (CAS, 11 items)

Cultural competence behaviors (CCB, 14 items)

Self-assessment

67 Items with 3 subscales (4-point Likert scale)

Knowledge of Diverse Patient Populations (23 items)

Adapting Health Care for Diverse Patient Populations Research study with healthcare

(21 items)

Promoting the Health of Diverse Communities (23 items)

Self-assessment

63 items with 4 domains (5-point Likert scale):

Knowledge (16 items)

Skills (15 items)

Attitudes (20 items)

Encounters (12 items)

\section{Self-assessment}

48 items with 5 scales (5- and 7-point Likert scales):

Awareness and sensitivity (11 items)

Behaviors (16 items)

Patient-centered communication (3 items)

Practice orientation (9 items)

Self-assessment (9 items)

Self-assessment

25 items with 5 subscales (4-point Likert):

Cultural awareness (5 items)
Studies $^{\mathrm{a}}$

Validation study with pharmacy

students ${ }^{44}$

Research study with pharmacists ${ }^{37}$

Validation study with healthcare professionals $^{31}$

students $^{32}$

Validation study with pharmacy

students ${ }^{13}$

Research studies with pharmacy students $^{20-23}$

Validation study with healthcare professionals $^{33}$

Validation studies with healthcare students ${ }^{19}$ 
Healthcare Professionals Revised (IAPCC-R)

Assessing the Process of Cultural Competence Among Healthcare Professionals (IAPCC). A student version (IAPCC-SV) is also available which uses a subset of 20 items.

Self-Assessment of Perceived Level of Cultural Competence (SAPLCC) and Revised SAPLCC
A combination of two assessmentsthe Clinical Cultural Competency Questionnaire

(CCCQ) and the California Brief Multicultural Competency Scale (CBMCS). The revised version uses 75 of the 86 items from the original version.
Cultural knowledge (5 items)

Cultural skill (5 items)

Cultural encounters (5 items)

Cultural desire (5 items)

Self-assessment

75 items with 6 domains (4-point Likert scale):

Knowledge (16 items)

Skills (11 items)

Attitudes (15 items)

Encounters (11 items)

Abilities (13 items)

Awareness (9 items)
Research studies with pharmacy students ${ }^{18,24-26}$

\section{Assessment Tools for Broad Student Use}

Everyday Multicultural

Competencies/Revised SEE (EMC/RSEE)

(ISS)

Multicultural Experiences Questionnaire (MEQ)
Developed from four previously

developed tools to assess

multicultural knowledge. These four tools were: Scale of Ethnocultural

Empathy, Miville-Guzman

Universality Diversity Scale-Short

Form, Openness to

Diversity/Challenge Scale, and Balanced Inventory of Desired Responding.

\section{Measures level of cultural} sensitivity. Validated with students in communication basic courses and was applied to pharmacy students in recent study.

Measures depth and breadth of multicultural experiences, along with desire to learn about and accommodate the views of others.

\section{Self-assessment}

48 items with 6 subscales $(5,6$, or 7-point Likert scale):

Cultural openness and desire to learn (10 items)

Resentment and cultural dominance (10 items)

Anxiety and lack of multicultural self-efficacy (7 items)

Empathic perspective-taking (5 items)

Awareness of contemporary racism and privilege (8

items)

Empathic feeling and acting as an ally (8 items)

Self-assessment

24 items with 5 scales (6-point Likert scale): Interaction engagement (7 items)

Respect for cultural differences (6 items)

Interaction confidence (5 items)

Interaction enjoyment ( 3 items)

Interaction attentiveness (3 items)

Self-assessment (5-point Likert scale):

15 items

Experience (number of items not reported)

Desire (number of items not reported)
Validation studies with pharmacy students ${ }^{13,48}$

Research studies with pharmacy students 27,28

Research study with medical students $^{27}$
Validation study with undergraduates in introductory psychology courses $^{14}$

Research study with medical students $^{34}$
Validation study with students in communication basic courses ${ }^{49}$

Research study with pharmacy students $^{29}$

Validation study with undergraduates ${ }^{50}$

Research study with physician assistant students ${ }^{35}$ 
Figure 1. Cultural Competence Tool Publication Search and Selection

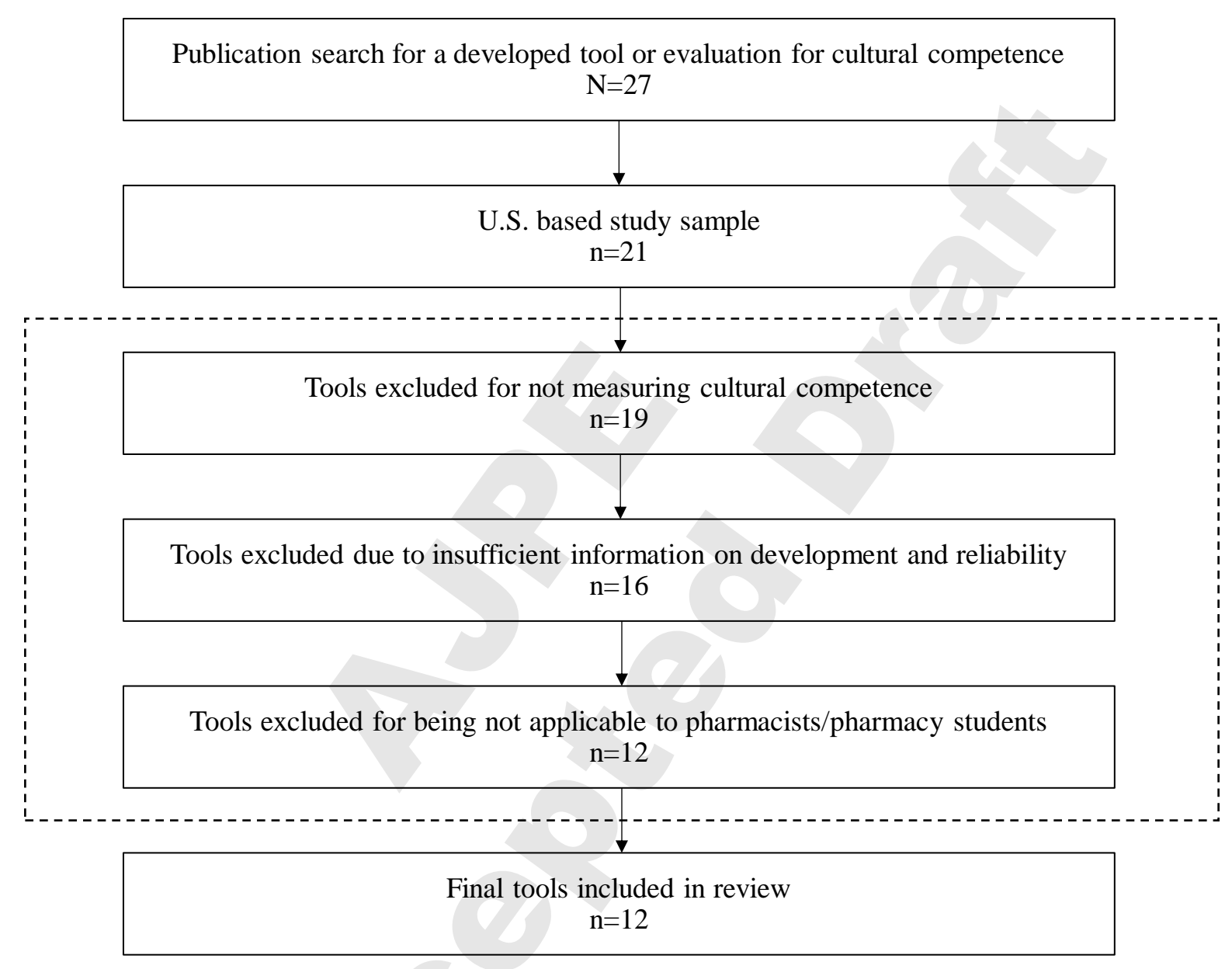

